Original article

\title{
Effects of rosuvastatin (added to hypocaloric diet) on serum periostin, adiponectin, proinflammtory cytokines levels and hepatic steatosis in non-alcoholic fatty liver disease patients with dyslipidemia
}

\author{
Rashid Ali Khan ${ }^{\mathrm{a}, *}$, Uma Bhandari ${ }^{\mathrm{b}}$, Prem Kapurc ${ }^{\mathrm{c}}$, Abhinav Jain ${ }^{\mathrm{d}}$, Farrukh Farah ${ }^{\mathrm{e}}$ \\ a Department of Pharmaceutical Medicine, School of Pharmaceutical Education and Research, Jamia Hamdard, New Delhi, 110062, India \\ ${ }^{\mathrm{b}}$ Department of Pharmacology, School of Pharmaceutical Education and Research, Jamia Hamdard, New Delhi, 110062, India \\ ${ }^{\mathrm{c}}$ Department of Medicine, Hamdard Institute of Medical Sciences \& Research, Jamia Hamdard, New Delhi, India \\ d Department of Radiology, Hamdard Institute of Medical Sciences E Research, Jamia Hamdard, New Delhi, India \\ e Department of Paramedical Sciences, Hamdard Institute of Medical Sciences E Research, Jamia Hamdard, New Delhi, India
}

A R T I C L E I N F O

\section{Article history:}

Received 25 November 2017

Received in revised form 23 December 2017

Accepted 29 December 2017

Available online 5 January 2018

\section{Keywords:}

Rosuvastatin

Non-alcoholic fatty liver disease

Dyslipidemia

Hepatic steatosis

Periostin

\begin{abstract}
A B S T R A C T
Objective: Non-alcoholic fatty liver disease (NAFLD) is strongly associated with dyslipidemia. Rosuvastatin exhibits greater efficacy in the treatment of dyslipidemia as compared to other statins, so we aimed to explore its effect on NAFLD patients.

Methods: We conducted a prospective observational study (6-month) to evaluate NAFLD patients with dyslipidemia receiving rosuvastatin with hypocaloric diet $(N=81)$ or hypocaloric diet alone $(N=114)$. Using propensity score matching, patients $(\mathrm{N}=76)$ were divided into two equal groups. Serum levels of periostin, adiponectin, TNF $\alpha$ and IL- 6 were determined using ELISA kits; hepatic steatosis was evaluated by ultrasound; hepatic fibrosis was assessed by NAFLD fibrosis and BARD scores at 0 and 6 months, and liver enzymes were assessed at 0, 2, 4, 6 months of study treatment.

Results: Rosuvastatin, in addition to hypocaloric diet diminished hepatic steatosis $(\mathrm{p}=0.011)$; decreased serum periostin $(p=0.003)$, TNF $\alpha(p=0.012)$, IL-6 levels $(p=0.010)$; increased serum adiponectin levels $(p=0.028)$, and improved NFS, BARD scores $(p>0.05)$. Alteration in grades of hepatic steatosis were found significantly correlated with change in serum levels of periostin $(r s=0.256, p=0.025)$ and adiponectin ( $r s=0.282, p=0.014$ ), while association was insignificant with the changes in serum TNF- $\alpha$ ( $\mathrm{rs}=0.148, \mathrm{p}=0.202$ ) and IL-6 levels ( $\mathrm{rs}=0.127, \mathrm{p}=0.274)$.

Conclusion: Treatment with rosuvastatin, in addition to the hypocaloric diet, resulted in a significant decrease in serum periostin levels; ameliorated grades of hepatic steatosis and fibrosis scores, indicating that rosuvastatin, in addition to hypocaloric diet may be effective treatment for NAFLD with dyslipidemia.
\end{abstract}

C 2017 Published by Elsevier, a division of RELX India, Pvt. Ltd on behalf of INDIACLEN.

\section{Introduction}

Non-alcoholic fatty liver disease (NAFLD) is the most common chronic liver disease encircling a histological scale progressing from simple steatosis to steatohepatitis, advanced fibrosis and cirrhosis. NAFLD is strongly associated with obesity, insulin resistance, dyslipidemia and oxidative stress. ${ }^{1}$ Approximately $60 \%$ of patients with dyslipidemia had evidence of hepatic steatosis while $70 \%$ of patients with non-alcoholic steatohepatitis (NASH) were found to have dyslipidemia. ${ }^{2}$

\footnotetext{
* Corresponding author.

E-mail address: rakhan.sch@jamiahamdard.ac.in (R.A. Khan).
}

Till date, there is no approved and established treatment for NAFLD. Aggressive treatment of dyslipidemia may reverse or stabilise NAFLD. Although statins might show efficacy in NAFLD due to their lipid-lowering function and pleiotropic effects but rare possibility of association of statins with hepatotoxicity, de novo cholesterol and fatty acid synthesis, ${ }^{3}$ limits its use in the treatment of NAFLD.

Rosuvastatin, a 3-hydroxy-3-methylglutaryl- coenzyme A (HMG-CoA) reductase inhibitor, exhibits greater efficacy in the treatment of dyslipidemia due to low lipophilicity, greater binding with HMG-CoA reductase enzyme, higher hepatocyte selectivity, minimal metabolism and low propensity for the hepatic cytochrome P450 (CYP) drug interactions as compared with other statins. ${ }^{4}$ 
A recent clinical study suggested Periostin, a novel matricellular protein, as a potential biomarker in the management of NAFLD. ${ }^{5}$ Periostin plays an important role in the progression of steatohepatitis, inflammation, and fibrosis in NAFLD. ${ }^{6-8}$ No treatment modalities have focussed to downregulate serum periostin level as a therapeutic strategy for treatment of NAFLD. In an animal study, Rosuvastatin inhibited periostin level and showed improvement in fibrosis ${ }^{9}$ but clinical relevance is not reported yet.

Clinical studies assessing the efficacy of rosuvastatin in NAFLD is limited and not well evidenced despite of its potential candidature in the treatment of NAFLD patients. Therefore, this study was undertaken to examine the effect of rosuvastatin on hepatic steatosis, periostin and find out its correlation. Additionally, study group also aimed to explore the effect of rosuvastatin on adiponectin, TNF- $\alpha$ and IL-6, and their correlation with grades of hepatic steatosis for reviewing rationalised prescription of rosuvastatin in NAFLD patients with dyslipidemia.

\section{Material and methods}

\subsection{Study design}

This 6-month, prospective observational study was conducted over the period between April 2015 to January 2017 in the clinical setting at Hamdard Institute of Medical Sciences \& Research (HIMSR), Jamia Hamdard, New Delhi, India. The study protocol was approved by Jamia Hamdard Institutional Ethics Committee (JHIEC), India (Registration no: ECR/162/Jamia/Inst/DL/2013). This study was carried out in accordance with the principles enunciated in the Declaration of Helsinki (Brazil, October, 2013). ${ }^{10}$ This study strictly followed the statement for strengthening the reporting of observational studies in epidemiology (STROBE). ${ }^{11}$ Patients were enrolled in this study only after receiving their written informed consent form.

\subsection{Subject selection}

The newly diagnosed NAFLD patients with dyslipidemia receiving treatments (rosuvastatin, $10 \mathrm{mg}$, once in a day with hypocaloric diet or hypocaloric diet only) were recruited in the study. Inclusion criteria were NAFLD patients taking study treatments of either sex of age of 18 to 75 years; BMI of 21 to 35; fasting plasma TG levels of 1.8 to $8 \mathrm{mmol}$ per liter or a ratio of total cholesterol to HDL cholesterol higher than 5 (among men) and higher than 4.5 (among women), or both. Patients were excluded for any of the following reasons: pregnant or nursing women; a history of pharmacological treatments of dyslipidemia and weight loss or consumption of other steatosis-causing drugs such as calcium channel blockers, tamoxifen, amiodarone, prednisolone, corticosterone and methotrexate; history of drug abuse; medical history of asthma, serious gastrointestinal, heart, kidney, mental and bone diseases; any history of surgery within the previous 6 months; patients with a known cause for their increased liver enzyme levels such as viral hepatitis (B or C); history of alcohol consumption of $>20 \mathrm{~g} /$ day in the past 2 years and recent changes in smoking habits. Based on the intervention, the patients were grouped in the study, either into rosuvastatin treatment group (rosuvastatin with hypocaloric diet) or diet control group (hypocaloric diet alone). Intake of rosuvastatin was confirmed by prescription as well as through patients' interview during their clinic visits.

\subsection{Diet and behaviour modifications}

Both physician and dietician educated all patients about eating behaviour and other adverse habits. Daily calorie requirement was calculated using revised Harris-Benedict equation. ${ }^{12}$ Patients were counselled to follow an individualised diet plan structured in accordance with their hypocaloric diet requirement. Each hypocaloric diet chart was composed of nutrients providing total energy as $55 \%$ carbohydrate, $15 \%$ protein, and $30 \%$ fat. Patients were asked to record a 7-day dietary intake and 24-h dietary recall which are monitored once in a month during their clinic visits or were enquired telephonically.

\subsection{Clinical and biochemical assessment and follow-up}

Demographic characteristics, anthropometric measurements and biochemical parameters including periostin, adiponectin, TNF$\alpha$, IL-6, total cholesterol (TC), triglycerides (TG), low-density lipoprotein (LDL) cholesterol, high-density lipoprotein (HDL) cholesterol, fasting blood glucose and insulin levels, glycated haemoglobin (HbA1c), complete blood count, bilirubin at 0 and 6 months; alanine aminotransferase (ALT), aspartate aminotransferase (AST), alkaline phosphatase (ALP) levels at 0, 2, 4 and 6 months of study treatments were determined using ELISA kits at pathology centre, HIMSR.

Homeostasis model assessment (HOMA) index was calculated at 0 and 6 months from the following equation: HOMA index = [fasting plasma glucose level $(\mathrm{mmol} / \mathrm{l}) \times$ fasting plasma insulin level $(\mu \mathrm{U} / \mathrm{ml})] / 22 . .^{13}$

\subsection{Chemicals}

Elisa kits of lipid profile, blood glucose levels, HbA1c, liver enzymes, bilirubin from Siemens Healthcare, Frimley, Camberley, UK; Insulin kits from Roche Diagnostics, Indianapolis, Germany; Periostin, TNF- $\alpha$, and IL-6 ELISA kits from Sincere Biotech, China; Adiponectin ELISA kits from Assaypro, USA, were utilised.

\subsection{Imaging study}

Hepatic steatosis was determined by assessing ultrasound (US) echogenicity of liver, using a single system GE Voluson S6 ultrasound machine with $4 \mathrm{C}$ wide frequency convex transducer, following not less than eight hours fasting at 0 and after 6 months of study treatments by an expert radiologist, who is unaware of the objectives of the study. The presence or absence and grading of hepatic steatosis were recorded according to standard structure documented in Goldberg textbook. ${ }^{14}$ urrent study utilised working clinical definition of NAFLD as: (i) grades of liver imaging meet the diagnostic criteria of fatty liver that cannot be explained by any other causes; or (ii) an unexplained steady rise in serum ALT, AST levels for at least 6 months in patients with any component of the metabolic syndrome. ${ }^{15}$

\subsection{NAFLD fibrosis score (NFS) and BARD score}

NAFLD fibrosis score (NFS) and BARD score were calculated for identification of advanced fibrosis at 0 and 6 months in both group by following equations: NFS $=-1.675+0.037$-age (years) +0.094 BMI $(\mathrm{kg} / \mathrm{m} 2)+1.13 \times \mathrm{IFG} /$ diabetes $($ yes $=1$, no $=0)+0.99 \times$ AST $/$ ALT ratio $-0.013 \times$ platelet count $(\times 109 / 1)-0.66 \times$ albumin $(\mathrm{g} / \mathrm{dl})$. A score lower than -1.455 excludes advanced fibrosis, a score higher than 0.676 indicates advanced fibrosis. Scores between these values are defined as indeterminate. ${ }^{16}$

The BARD score was determined by an addition of points of following three variables: AST/ALT ratio $\geq 0.8-2$ points; a BMI $\geq 28$ 1 point; and the presence of diabetes -1 point. A score equal to 0 or 1 predicts high (96\%) negative predictive value (NPV) for advanced fibrosis. ${ }^{17}$ 


\subsection{Sample size calculation}

Till date, no previous clinical study evaluated the effect of rosuvastatin on serum periostin levels. Hence, sample size was calculated on the basis of a pilot study, which evaluated the effect of rosuvastatin on serum periostin levels in pre and post design manner in 10 propensity score matched patients with $5 \% \alpha .30$ patients were required for $80 \%$ power, and 45 patients were required for $90 \%$ power of the study.

\subsection{Statistical analysis}

Descriptive statistics were used to analyse the demographic and clinical characteristics of the patients. Data were expressed as mean (SD) or median value (interquartile range), and normal distribution of variables were identified using KolmogorovSmirnov test. Independent sample $t$ tests were used for between-group comparisons at baseline. Paired t-tests were used to evaluate within-treatment changes from baseline to end of treatment whereas between-treatment changes at the end of treatment were tested using 1-way analysis of covariance with the initial value as a covariate. ANOVA was used for repeated measurements of liver enzymes. The Wilcoxon signed-rank test or the Mann-Whitney test was used if nonparametric tests were required. Spearman rho or pearson correlation analysis were used for correlation.

Propensity score analysis was conducted to minimise selection bias, resulted due to non-randomised nature of the study. The propensity score was computed using multivariate logistic regression analysis, taking covariates such as age, sex, BMI, smoking status, family history of obesity, presence of diabetes, grades of hepatic steatosis, fibrosis scales, TG levels, ratio of total cholesterol to HDL cholesterol. Treatment and control group patients were matched in $1: 1$ by using nearest neighbour propensity score matching method with a caliper of 0.05 units.

Data were analysed by using software of statistical package for the social sciences (SPSS, version 21.0, SPSS, Inc., Chicago, IL, USA). The power of sample size was calculated by G*Power (version 3.1, Heinrich-Heine-Universität, Düsseldorf, Germany).

\section{Results}

A total of 173 newly diagnosed NAFLD patients prescribed with rosuvastatin plus hypocaloric diet $(\mathrm{N}=78)$ or hypocaloric diet alone $(\mathrm{N}=95)$, were screened in accordance with study criteria. At the end of the study, only 111 patients $(N=53$, rosuvastatin group; $\mathrm{N}=58$, diet control group) completed the 6 month study period (detailed description given in Fig. 1). Mild gastrointestinal side effects, non-adherent to study drug, noncompliance with dietary restriction, financial reasons and unwillingness to visit the centre and commitment towards daily work in rosuvastatin group while switching to drug treatment or noncompliance with dietary restriction in the diet control group were the major reasons for non-completion of the study. Significant differences were found between treatment groups in baseline demographic, clinical, laboratory and ultrasound imaging characteristics (Tables 1 and 2). In accordance with propensity scores, 76 patients with matched scores were divided into two

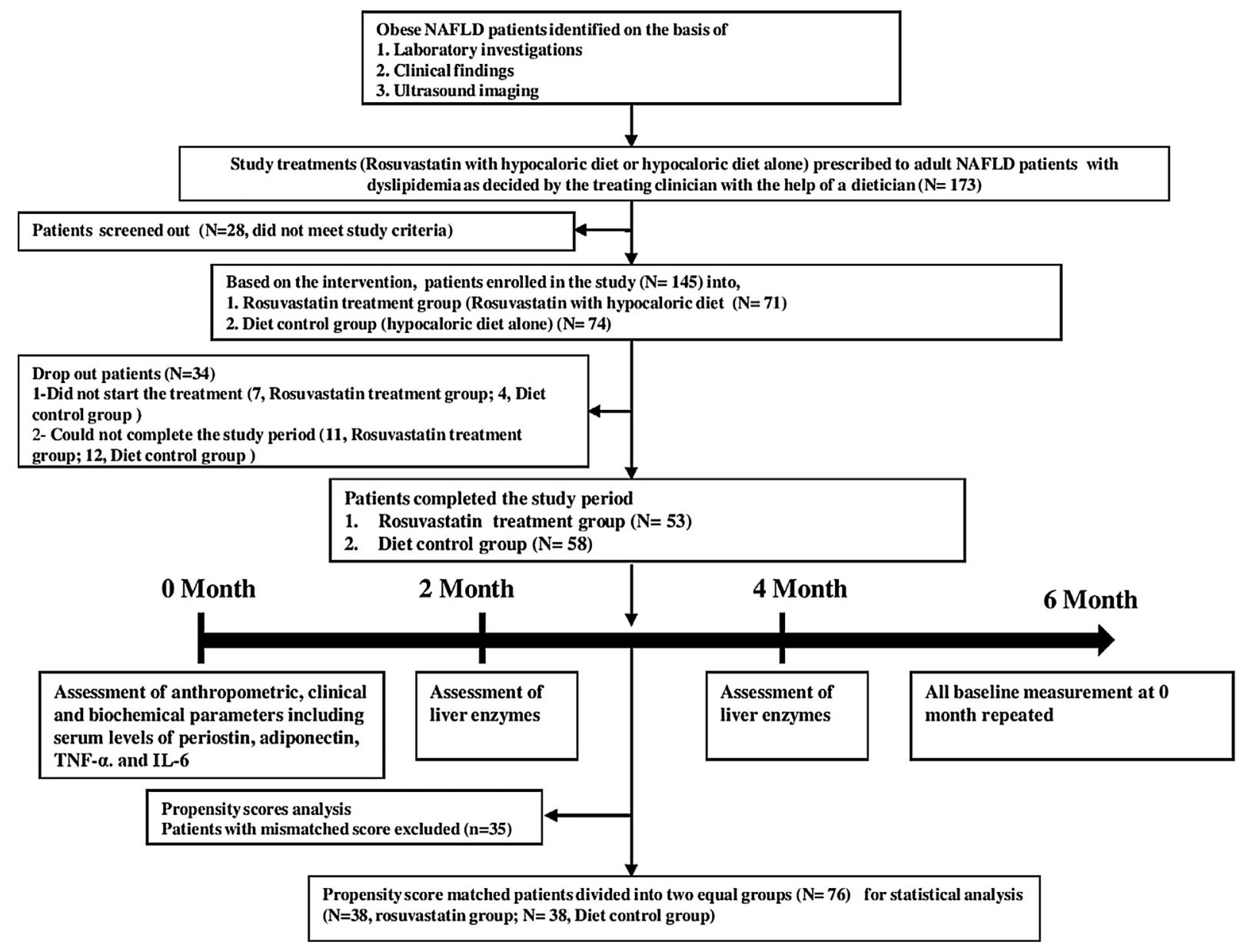

Fig. 1. Study design. 
Table 1

Baseline Demographics, Clinical and Laboratory Data.

\begin{tabular}{|c|c|c|c|c|c|c|}
\hline \multirow[t]{2}{*}{ Variable } & \multicolumn{3}{|c|}{ Propensity score matching $(-)$} & \multicolumn{3}{|c|}{ Propensity score matching $(+)$} \\
\hline & Control (58) & Rosuvastatin (53) & $P$ Value & Control (38) & Rosuvastatin (38) & $P$ Value \\
\hline Age (y) & $50.72 \pm 13.90$ & $44.78 \pm 10.80$ & $0.006^{*}$ & $44.57 \pm 11.19$ & $40.60 \pm 9.95$ & 0.128 \\
\hline Age $\geq 60$ & $14(24.14)$ & $7(13.21)$ & 0.116 & $8(21.05)$ & $5(13.16)$ & 0.367 \\
\hline Female sex & $30(51.72)$ & $22(41.50)$ & 0.184 & $15(39.48)$ & $18(47.37)$ & 0.494 \\
\hline DM & $13(22.41)$ & $8(15.09)$ & 0.376 & $7(18.42)$ & $4(10.53)$ & 0.335 \\
\hline Hypertension & $9(15.51)$ & $15(28.30)$ & $0.019 *$ & $6(15.79)$ & $11(28.95)$ & 0.173 \\
\hline History of obesity & $6(10.34)$ & $13(24.52)$ & $0.025^{*}$ & $4(10.53)$ & $7(18.42)$ & 0.335 \\
\hline Smoking & $14(24.14)$ & $6(11.32)$ & 0.189 & $9(23.68)$ & $4(10.53)$ & 0.132 \\
\hline Weight & $80.73 \pm 9.00$ & $74.76 \pm 6.92$ & $<0.001^{* *}$ & $77.90 \pm 8.85$ & $75.41 \pm 5.07$ & 0.137 \\
\hline BMI & $28.58 \pm 3.29$ & $26.56 \pm 2.38$ & $<0.001^{* *}$ & $27.80 \pm 3.44$ & $26.81 \pm 1.62$ & 0.118 \\
\hline $\mathrm{BMI} \geq 30$ & $19(32.76)$ & $6(11.32)$ & $0.008^{*}$ & $11(28.95)$ & $5(13.16)$ & 0.094 \\
\hline Waist (m) & $1.02 \pm .05$ & $0.99 \pm 0.03$ & $0.001^{*}$ & $0.99 \pm 0.04$ & $0.98 \pm 0.02$ & 0.502 \\
\hline Waist/hip ratio & $0.98 \pm 0.03$ & $0.96 \pm 0.02$ & $0.002^{*}$ & $0.97 \pm 0.01$ & $0.97 \pm 0.01$ & 0.108 \\
\hline $\mathrm{FPG}(\mathrm{mmol} / \mathrm{L})$ & $5.78 \pm 0.78$ & $5.44 \pm 0.74$ & $0.008^{*}$ & $5.34 \pm 0.77$ & $5.55 \pm 0.73$ & 0.234 \\
\hline HbA1c (\%) & $5.69 \pm 0.41$ & $5.55 \pm 0.35$ & $0.025^{*}$ & $5.48 \pm 0.24$ & $5.58 \pm 0.40$ & 0.153 \\
\hline Insulin (pmol/L) & $125.19 \pm 43.50$ & $108.19 \pm 30.34$ & $0.010^{*}$ & $104.34 \pm 19.02$ & $96.03 \pm 19.03$ & 0.067 \\
\hline HOMA-IR & $4.71 \pm 2.01$ & $3.74 \pm 1.05$ & $0.001^{*}$ & $3.57 \pm 0.82$ & $3.40 \pm 0.82$ & 0.380 \\
\hline $\mathrm{TC}(\mathrm{mmol} / \mathrm{L})$ & $5.35 \pm 1.06$ & $7.48 \pm 1.73$ & $<0.001^{* * *}$ & $6.67 \pm 1.19$ & $6.85 \pm 1.04$ & 0.487 \\
\hline $\mathrm{TG}(\mathrm{mmol} / \mathrm{L})$ & $2.30 \pm 0.51$ & $2.58 \pm 0.45$ & $0.001^{*}$ & $2.49 \pm 0.44$ & $2.62 \pm 0.43$ & 0.205 \\
\hline $\mathrm{LDL}(\mathrm{mmol} / \mathrm{L})$ & $4.63 \pm 1.02$ & $5.15 \pm 0.99$ & $<0.001^{* *}$ & $4.63 \pm 1.13$ & $4.82 \pm 0.98$ & 0.417 \\
\hline $\mathrm{HDL}(\mathrm{mmol} / \mathrm{L})$ & $0.88 \pm 0.06$ & $0.87 \pm 0.07$ & 0.160 & $0.90 \pm 0.06$ & $0.86 \pm 0.07$ & 0.091 \\
\hline ALT(ukat/L) & $0.92 \pm 0.32$ & $1.07 \pm 0.30$ & $0.005^{*}$ & $1.04 \pm 0.30$ & $1.09 \pm 0.30$ & 0.455 \\
\hline AST(ukat/L) & $0.61 \pm 0.24$ & $0.71 \pm 0.26$ & $0.038^{*}$ & $0.68 \pm 0.26$ & $0.75 \pm 0.27$ & 0.262 \\
\hline $\operatorname{ALP}($ ukat/L) & $1.66 \pm 0.27$ & $1.84 \pm 0.36$ & $0.001^{*}$ & $1.57 \pm 0.29$ & $1.57 \pm 0.30$ & 0.146 \\
\hline $\operatorname{Bilirubin}(\mu \mathrm{mol} / \mathrm{L})$ & $11.31 \pm 6.33$ & $12.20 \pm 7.40$ & 0.440 & $8.63(5.64-18.16)$ & $12.48(6.62-17.01)$ & 0.332 \\
\hline Periostin (ng/ml) & $1605.72 \pm 502.14$ & $1934.86 \pm 484.57$ & $<0.001^{* *}$ & $1856.72 \pm 541.50$ & $2032.86 \pm 451.71$ & 0.128 \\
\hline Adiponectin $(\mu \mathrm{g} / \mathrm{ml})$ & $4.28 \pm 0.98$ & $4.62 \pm 1.04$ & 0.051 & $4.80 \pm 1.02$ & $4.49 \pm 1.06$ & 0.205 \\
\hline $\mathrm{TNF} \alpha(\mathrm{pg} / \mathrm{ml})$ & $6.57 \pm 1.70$ & $6.98 \pm 1.66$ & 0.153 & $6.70 \pm 1.52$ & $7.32 \pm 1.65$ & 0.087 \\
\hline IL-6 (pg/ml) & $3.88 \pm 1.75$ & $4.38 \pm 1.54$ & 0.072 & $4.20 \pm 1.70$ & $4.82 \pm 1.29$ & 0.079 \\
\hline
\end{tabular}

Data are mean $\pm \mathrm{SD}, \mathrm{n}(\%)$, median (interquartile range).

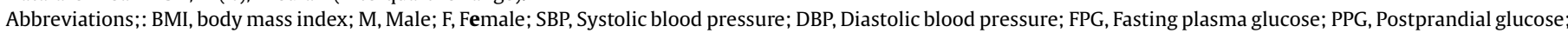

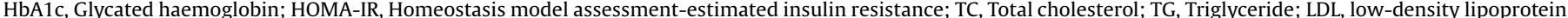

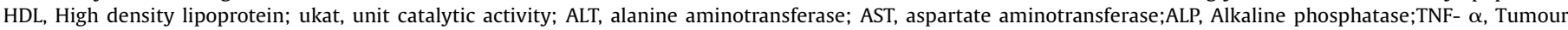
necrosis factor alpha; IL-6, Interleukin-6.

${ }^{*} \mathrm{p}<0.05$, value significantly different between groups.

$\mathrm{p}<0.001$, value significantly different between groups.

Table 2

Baseline ultrasound grading and fibrosis scores.

\begin{tabular}{|c|c|c|c|c|c|c|}
\hline \multirow[t]{2}{*}{ Variable } & \multicolumn{3}{|c|}{ Propensity score matching $(-)$} & \multicolumn{3}{|c|}{ Propensity score matching $(+)$} \\
\hline & Control (58) & Rosuvastatin (53) & $P$ Value & Control (38) & Rosuvastatin (38) & $P$ Value \\
\hline US grades & $1.65 \pm 0.71$ & $1.96 \pm 0.62$ & $0.012^{*}$ & $1.84 \pm 0.71$ & $2.00 \pm 5.07$ & 0.303 \\
\hline 0 & 0 & 0 & & 0 & 0 & \\
\hline 1 & $28(48.27)$ & $11(20.75)$ & & $13(34.21)$ & $8(21.05)$ & \\
\hline 2 & $22(37.93)$ & $33(62.26)$ & & $18(47.37)$ & $22(57.89)$ & \\
\hline 3 & $8(13.79)$ & $9(16.98)$ & & $7(18.42)$ & $8(21.05)$ & \\
\hline NFS score & $1.33 \pm 0.47$ & $1.21 \pm 0.41$ & 0.157 & $1.31 \pm 0.62$ & $1.16 \pm 0.40$ & 0.322 \\
\hline Low & $39(67.24)$ & $42(79.24)$ & & $29(76.32)$ & $32(84.21)$ & \\
\hline Intermediate & $19(32.75)$ & $11(20.75)$ & & $9(23.68)$ & $6(15.79)$ & \\
\hline High & 0 & 0 & & 0 & 0 & \\
\hline BARD score & $1.43 \pm 0.50$ & $1.30 \pm 0.46$ & $0.161^{*}$ & $1.37 \pm 0.99$ & $1.00 \pm 1.04$ & 0.106 \\
\hline Low & $33(56.90)$ & $37(69.81)$ & & $26(68.42)$ & $24(63.16)$ & \\
\hline High & $25(43.10)$ & $16(30.19)$ & & $12(31.58)$ & $14(36.84)$ & \\
\hline
\end{tabular}

Data are mean $\pm \mathrm{SD}, \mathrm{n}(\%)$.

Abbreviations: USultrasound; NFSNAFLD fibrosis score; BARDBody mass index plus AST/ALT ratio plus presence of diabetes.

$\mathrm{p}<0.05$, value significantly different between groups.

equal groups ( $\mathrm{n}=38$, rosuvastatin group; $\mathrm{n}=38$, diet control) and accounted for statistical analysis (Fig. 1). No significant differences were found in baseline characteristics, between propensity score matched treatment groups (Tables 1 and 2).

All enrolled subjects, included in the final analysis were adult (diet control vs. rosuvastatin group; mean age, $44.57 \pm 11.19$ year vs. $40.60 \pm 9.95$ year $)$ with mean BMI ( $27.80 \pm 3.44$ vs. $26.81 \pm 1.62)$ NAFLD patients. A total of $47.37 \%$ of the rosuvastatin group were women compared with $39.48 \%$ women in the control group. (Table 3)

\subsection{Treatment outcome}

\subsubsection{Clinical and biochemical characteristics}

At the end of the study, rosuvastatin group experienced insignificant reductions in weight, BMI and waist circumference, but significant improvements were observed in serum levels of total cholesterol, LDL cholesterol, TG, HDL cholesterol; plasma insulin levels and HOMA-IR values, as compared to diet control group (Table 3 ). Both groups showed insignificant changes in serum levels of AST, ALT, ALP, but mild rise ( $2>$ ULN) in AST and ALT levels in 6 patients (15.79\%) after 2 month treatment and ALP levels 
Table 3

Comparison of Pre- and Post clinical and Laboratory Variables within and between Groups among propensity matched patients.

\begin{tabular}{|c|c|c|c|c|c|c|c|}
\hline \multirow[t]{2}{*}{ Variable } & \multicolumn{2}{|l|}{ Control (38) } & \multirow[t]{2}{*}{ P value } & \multicolumn{2}{|l|}{ Rosuvastatin (38) } & \multirow[t]{2}{*}{$P$ value } & \multirow[t]{2}{*}{ P value Between groups } \\
\hline & 0 month & 6 months & & 0 month & 6 months & & \\
\hline Body weight (kg) & $77.90 \pm 8.85$ & $75.74 \pm 7.81$ & $<0.001^{* *}$ & $75.41 \pm 5.07$ & $73.63 \pm 5.25$ & $<0.011^{*}$ & 0.470 \\
\hline BMI $(\mathrm{kg} / \mathrm{m} 2)$ & $27.80 \pm 3.44$ & $26.81 \pm 3.03$ & $<0.001^{* *}$ & $26.81 \pm 1.62$ & $26.19 \pm 1.88$ & $0.012^{*}$ & 0.407 \\
\hline Waist (m) & $0.99 \pm 0.04$ & $0.98 \pm 0.03$ & $0.006^{*}$ & $0.98 \pm 0.02$ & $0.97 \pm 0.02$ & $0.022^{*}$ & 0.933 \\
\hline Waist/hip ratio & $0.97 \pm 0.01$ & $0.96 \pm 0.02$ & 0.186 & $0.97 \pm 0.01$ & $0.96 \pm 0.02$ & 0.386 & 0.882 \\
\hline FPG $(\mathrm{mmol} / \mathrm{L})$ & $5.34 \pm 0.77$ & $5.28 \pm 0.8$ & 0.575 & $5.55 \pm 0.73$ & $5.29 \pm 0.69$ & $0.004^{*}$ & 0.257 \\
\hline HbA1c (\%) & $5.48 \pm 0.24$ & $5.44 \pm 0.27$ & 0.160 & $5.58 \pm 0.40$ & $5.45 \pm 0.39$ & 0.013 & 0.215 \\
\hline Insulin (pmol/L) & $104.34 \pm 19.02$ & $100.028 \pm 14.51$ & 0.191 & $96.03 \pm 19.90$ & $87.54 \pm 21.24$ & $0.011^{*}$ & $0.024^{\dagger}$ \\
\hline HOMA-IR & $3.57 \pm 0.82$ & $3.40 \pm 0.82$ & 0.187 & $3.40 \pm 0.82$ & $2.96 \pm 0.81$ & $0.003^{*}$ & $0.032^{\dagger}$ \\
\hline $\mathrm{TC}(\mathrm{mmol} / \mathrm{L})$ & $6.67 \pm 1.19$ & $6.49 \pm 1.43$ & 0.180 & $6.85 \pm 1.04$ & $5.09 \pm 0.86$ & $<0.001^{* *}$ & $<0.001^{\dagger \dagger}$ \\
\hline $\mathrm{TG}(\mathrm{mmol} / \mathrm{L})$ & $2.49 \pm 0.44$ & $2.43 \pm 0.42$ & 0.334 & $2.62 \pm 0.43$ & $1.81 \pm 0.36$ & $<0.001^{* *}$ & $<0.001^{\dagger \dagger}$ \\
\hline $\mathrm{LDL}(\mathrm{mmol} / \mathrm{L})$ & $4.63 \pm 1.13$ & $4.45 \pm 1.36$ & 0.145 & $4.82 \pm 0.98$ & $3.27 \pm 0.36$ & $<0.001^{* *}$ & $<0.001^{\dagger \dagger}$ \\
\hline $\mathrm{HDL}(\mathrm{mmol} / \mathrm{L})$ & $0.90 \pm 0.06$ & $0.92 \pm 0.13$ & 0.326 & $0.86 \pm 0.07$ & $0.97 \pm 0.05$ & $<0.001^{* *}$ & $0.033^{\dagger}$ \\
\hline ALT(ukat/L) & $1.04 \pm 0.30$ & $0.98 \pm 0.23$ & 0.067 & $1.09 \pm 0.30$ & $1.02 \pm 0.24$ & 0.302 & 0.057 \\
\hline AST(ukat/L) & $0.68 \pm 0.26$ & $0.62 \pm 0.19$ & 0.065 & $0.75 \pm 0.27$ & $0.65 \pm 0.23$ & $0.122^{*}$ & 0.094 \\
\hline ALP(ukat/L) & $1.57 \pm 0.29$ & $154 \pm 0.27$ & 0.608 & $1.57 \pm 0.30$ & $1.50 \pm 0.36$ & 0.341 & 0.255 \\
\hline $\operatorname{Bilirubin}(\mu \mathrm{mol} / \mathrm{L})$ & 8.63(5.64-18.16) & $11.80(8.63-17.74)$ & 0.201 & $12.48(6.62-17.01)$ & $14.28(10.99-17.44)$ & 0.083 & 0.194 \\
\hline Periostin (ng/ml) & $1856.72 \pm 541.50$ & $1866.51 \pm 613.02$ & 0.758 & $2032.86 \pm 451.71$ & $1809.75 \pm 338.39$ & $0.003^{*}$ & $0.017^{\dagger}$ \\
\hline Adiponectin $(\mu \mathrm{g} / \mathrm{ml})$ & $4.80 \pm 1.02$ & $4.83 \pm 1.28$ & 0.798 & $4.49 \pm 1.06$ & $4.79 \pm 0.95$ & $0.028^{*}$ & 0.247 \\
\hline $\mathrm{TNF} \alpha(\mathrm{pg} / \mathrm{ml})$ & $6.70 \pm 1.52$ & $6.74 \pm 1.53$ & 0.577 & $7.32 \pm 1.65$ & $6.75 \pm 2.28$ & $0.012^{*}$ & $0.008^{\dagger}$ \\
\hline IL-6 (pg/ml) & $4.20 \pm 1.70$ & $4.31 \pm 1.53$ & 0.470 & $4.82 \pm 1.29$ & $4.21 \pm 1.60$ & $0.010^{*}$ & $0.039^{\dagger}$ \\
\hline
\end{tabular}

Data are mean $\pm \mathrm{SD}$, or median (interquartile range).

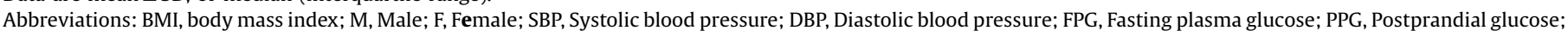

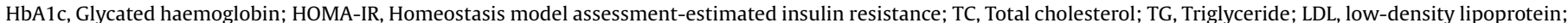

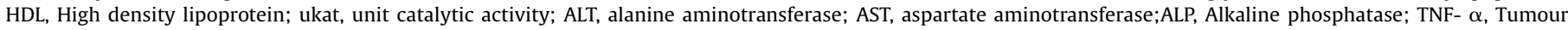
necrosis factor alpha; IL-6, Interleukin-6.

${ }^{*} \mathrm{P}$ value is less than 0.05 , within groups.

** P value is less than 0.001 within groups.

${ }^{\dagger} \mathrm{P}$ value is less than 0.05 between groups.

${ }^{\dagger} \mathrm{P}$ value is less than 0.001 between groups.

$(2>\mathrm{ULN})$ in 5 patients (13.16\%) after 4 month treatment, were also reported in rosuvastatin group but treatment was continued for 6 months, and at that moment mild rise in AST, ALT and ALP was still seen in 4 patients $(10.53 \%)$.

\subsubsection{Serum periostin, adiponectin, TNF $\alpha$ and $I L-6$}

After 6 months of treatment, rosuvastatin group demonstrated significant reductions in serum levels of periostin, TNF $\alpha$, IL-6, and increases in serum adiponectin levels. Diet control group also showed slight increase in serum adiponectin level but these changes did not reach statistical significance. Moreover, changes in serum levels of periostin, TNF $\alpha$ and IL-6 level were also not significant in the diet control group.
In comparison to hypocaloric diet group, rosuvastatin group also showed notable reductions in serum levels of periostin, TNF $\alpha$ and IL-6, but adiponectin levels were not increased significantly. (Table 3)

\subsubsection{Ultrasound imaging studies}

Rosuvastatin group caused a significant reversal of hepatic steatosis and showed diminished hepatic ultrasound echogenicity ( $p=0.011$ ) but this reduction did not reach statistical significance in comparison to the diet control group ( $p$ 0.255). Rosuvastatin group experienced improvement in steatosis in 18 patients as compared to 12 patients in diet control group. Furthermore, Fatty liver progressed in six patients of rosuvastatin group as compared to eight patients of diet control group. (Table 4)

Table 4

Comparison of NAFLD US grades, NFS and BARD score Within and Between Groups.

\begin{tabular}{|c|c|c|c|c|c|c|c|}
\hline \multirow[t]{2}{*}{ Variable } & \multicolumn{2}{|c|}{ Control (38) } & \multirow[t]{2}{*}{$P$ value } & \multicolumn{2}{|c|}{ Rosuvastatin (38) } & \multirow[t]{2}{*}{$P$ value } & \multirow[t]{2}{*}{$P$ value Between groups } \\
\hline & 0 month & 6 months & & 0 months & 6 months & & \\
\hline US grades & $1.84 \pm 0.71$ & $1.74 \pm 0.72$ & 0.437 & $2.00 \pm 5.07$ & $1.60 \pm 0.97$ & $0.011^{*}$ & 0.255 \\
\hline 0 & 0 & $2(5.26)$ & & 0 & $6(15.79)$ & & \\
\hline 1 & $13(34.21)$ & $10(26.31)$ & & $8(21.05)$ & $10(26.32)$ & & \\
\hline 2 & $18(47.37)$ & $22(57.90)$ & & $22(57.89)$ & $15(39.47)$ & & \\
\hline 3 & $7(18.42)$ & $4(10.53)$ & & $8(21.05)$ & $7(18.42)$ & & \\
\hline NFS score & $1.31 \pm 0.62$ & $1.45 \pm 0.76$ & 0.194 & $1.16 \pm 0.40$ & $1.02 \pm 0.16$ & $0.025^{*}$ & $0.003^{\dagger}$ \\
\hline Low & $29(76.32)$ & $27(71.05)$ & & $32(84.21)$ & $37(97.37)$ & & \\
\hline Intermediate & $9(23.68)$ & $11(28.95)$ & & $6(15.79)$ & $1(2.63)$ & & \\
\hline High & 0 & 0 & & 0 & 0 & & \\
\hline BARD score & $1.37 \pm 0.99$ & $1.29 \pm 0.77$ & 0.432 & $1.00 \pm 1.04$ & $0.60 \pm 0.85$ & $0.040^{*}$ & $0.002^{\dagger}$ \\
\hline Low & $26(68.42)$ & $28(73.68)$ & & $24(63.16)$ & $31(81.58)$ & & \\
\hline High & $12(31.58)$ & $10(26.31)$ & & $14(36.84)$ & $7(18.42)$ & & \\
\hline
\end{tabular}

Data are mean $\pm \mathrm{SD}, \mathrm{n}(\%)$.

Abbreviations: US, ultrasound; NFS, NAFLD fibrosis score; BARD, Body mass index plus AST/ALT ratio plus presence of diabetes.

" P value is less than 0.05 within groups.

$\dagger$ P value is less than 0.05 between groups. 


\subsubsection{NAFLD fibrosis score and BARD score}

After 6 months of treatment, rosuvastatin group showed a significant improvement in NFS and BARD score in comparison to diet control group $(\mathrm{p}<0.05)$. Further, hypocaloric diet group failed to improve NFS and BARD score $(\mathrm{p}>0.05)$. (Table 4$)$

\subsubsection{Correlation analysis}

Taking all patients into account, degree of reductions in ultrasound grade were found to be significantly correlated with decrease in serum periostin levels ( $r s=0.256, p=0.025$ ); increase in serum adiponectin levels ( $\mathrm{rs}=0.282, \mathrm{p}=0.014$ ) but association was found insignificant with changes in the serum levels of TNF- $\alpha$ ( $\mathrm{rs}=0.148, \mathrm{p}=0.202$ ) and IL-6 ( $\mathrm{rs}=0.127, \mathrm{p}=0.274)$. (Table 5)

\section{Discussion}

The present study evaluated the effect of 6-month rosuvastatin treatment along with hypocaloric diet on clinical, biochemical and radiological features of disease activity in NAFLD patients with dyslipidemia. This study demonstrated a significant reversal of fatty infiltration in liver with consequent improvement of NFS and BARD scores in rosuvastatin group while, hypocaloric diet alone could not ameliorate hepatic steatosis and failed to improve NFS and BARD scores.

The pleiotropic effects and lipid lowering property of rosuvastatin have been thought to be a possible reason for its therapeutic efficacy in NAFLD patients. Recently, an animal study by Souza Marinho et al (2016) demonstrated that rosuvastatin diminishes the steatosis through reduced lipogenesis by inhibiting PPAR$\gamma /$ SREBP-1c pathway. They, further, revealed that rosuvastatin increases $\beta$-oxidation of fatty acid through upregulation of PPAR$\alpha$. It has been reported that inhibition of SREBP-1c with rosuvastatin, ameliorates insulin resistance. In addition, they also demonstrated that expression of FAT/CD36, a transporter of fatty acid in human and animal hepatocytes, was diminished and fibrosis was prevented through inhibited HSCs activation. ${ }^{18}$

Oxidative stress also plays an important role in disease progression of NAFLD and, is associated with severity of hepatic steatosis. The pleiotropic antioxidant potential of rosuvastatin provides a valuable insight in favour of its beneficial effect in the treatment of NAFLD. ${ }^{19}$

Although, statins have been underused in NAFLD, due to the possibility of increased risk of hepatotoxicity, disturbed glucose homeostasis, and insulin resistance ${ }^{3}$, but the mechanisms regarding the detrimental effects of statins on NAFLD are still inconsistent. Conversely, few clinical studies have been reported that rosuvastatin might be effective in NAFLD patients with dyslipidemia. Antonopoulos and colleagues (2006) demonstrated a improved abnormal transaminases, and lipid profiles of NAFLD patients with 8 months of rosuvastatin treatment. ${ }^{20}$ Another clinical study also showed that rosuvastatin treatment alleviates the non-alcoholic steatohepatitis in patients with metabolic syndrome. ${ }^{21}$ A pilot study also reported the efficacy of rosuvastatin

Table 5

Correlation of degree of reduction in ultrasound score with percentage change in periostin, adiponectin, TNF- $\alpha$ and IL- 6 after 6 months treatment of Rosuvastatin.

\begin{tabular}{lll}
\hline Factor & $\mathrm{r}_{\mathrm{s}}$ & P value \\
\hline \% Periostin decrease & 0.256 & $0.025^{*}$ \\
\% Adiponectin increase & 0.282 & $0.014^{*}$ \\
\% TNF- $\alpha$ decrease & 0.148 & 0.202 \\
\% IL-6 decrease & 0.127 & 0.274 \\
\hline
\end{tabular}

Abbreviations;: $r_{s}$, spearman correlation; TNF- $\alpha$, Tumor necrosis factor alpha; IL-6, Interleukin-6.

$\mathrm{P}$ value is less than 0.05 . in NAFLD, however, the results were not so promising, and needed a large controlled study to establish the efficacy. ${ }^{22}$

In consistent with previous findings, the present study also demonstrated a insignificant changes in AST and ALT levels after 6month treatment with rosuvastatin. Further, we noted mild elevation in AST and ALT levels in 6 patients (15.79\%). Elevation of liver enzymes with statins could be due to transaminitis, which is not necessarily associated with hepatotoxicity and it might be possibly due to natural disease progression instead of statinrelated effect. ${ }^{23}$

McKenney et al (2005) reported that rosuvastatin is effective and safe in the treatment of dyslipidemia in diabetic and nondiabetic patients at therapeutic doses. ${ }^{24}$ The current study also observed a notable improvement in glucose homeostasis in rosuvastatin treated patients.

Statins may cause impaired glucose metabolism through the activation of NLRP3 inflammasome, which contribute to the development of Type 2 diabetes mellitus. ${ }^{25}$ Athyros et al (2017) suggested that physicians should consider use of statins in the treatment of NAFLD at high CVD or HCC risk in combination with pioglitazone. ${ }^{26}$

Luo B et al. (2014) reported inhibitory effects of rosuvastatin on NLRP3 inflammasome and MAPK pathways in the animal model. ${ }^{27}$ Although clinical relevance is still not established with rosuvastatin treatment, but it could be a possible mechanism for beneficial effect of rosuvastatin on blood glucose homeostasis in the present study.

Recent preclinical and clinical studies indicated a pivotal role of periostin in the progression of NAFLD i.e. steatohepatitis, inflammation, and fibrosis. Periostin play critically in the regulation of hepatic triglyceride homeostasis and possibly involved in insulin sensitivity. ${ }^{6-8,28}$ TGF- $\beta$ is a key stimulator of periostin secretion in a variety of tissues or cells and periostin expression is decreased by inhibition of TGF- $\beta{ }^{8,29}$ Interestingly, Ma et al. (2013) documented that rosuvastatin inhibits TGF- $\beta 1$ expression and alleviates myocardial fibrosis in diabetic rats. ${ }^{30}$ Another study also demonstrated the inhibitory effect of rosuvastatin on the expression of periostin in cardiac model of rat. ${ }^{9}$ In accordance with previous studies, the present study explored the effect of rosuvastatin on serum periostin levels and showed a significant reduction $(p=0.002)$ in serum periostin levels as compared to the hypocaloric diet in NAFLD patients.

A recent study on Chinese obese patients suggests that TG, TNF$\alpha$, IL-6 and HOMA-IR were independent factors influencing the periostin levels. $^{28}$ Treatment with TNF- $\alpha$ increases the mRNA expression of periostin in HepG2 cells. ${ }^{8}$ Hence, it is conceivable that inflammatory cytokines may involve in the regulation of periostin biosynthesis in target tissues. In the present study, improvement in the levels of TG, TNF- $\alpha$, IL- 6 and HOMA-IR with rosuvastatin might be responsible for downregulation of periostin levels.

Imbalance of adipocytokines (decreased serum adiponectin, increased serum TNF- $\alpha$ and IL-6 levels) were normalised after rosuvastatin treatment as compared to hypocaloric diet alone. This ameliorative effect of rosuvastatin might be due to the pleiotropic property of statins which is in line with the previous clinical study. $^{22}$

Significant positive association between the decreased serum levels of periostin and degree of reduction in NAFLD US grades ( $r s=0.256, p=0.025$ ) was also established in our study. Hence, the effect of rosuvastatin on grades of fatty liver might also be due to indirect downregulation of periostin.

We, further, noted the significant correlation of an increases in adiponectin levels with degree of reductions in NAFLD US grades ( $r s=0.282, p=0.014$ ) which is similar to the findings of the previous study. ${ }^{31}$ This study also supports the proposal by Finelli 
et al. (2013), that downregulation of adiponectin levels might be a therapeutic approach for the management of NAFLD. ${ }^{32}$

In the present study, adverse effects related to the gastrointestinal tract mainly constipation, stomach-ache, indigestion and diarrhoea were reported in $9(23.68 \%)$ patients and myalgia were also reported in 4 patients (10.52\%). Sleep problems were also reported in $2(5.26 \%)$ patients.

\subsection{Limitations}

The present study should be interpreted in the light of some limitations. Small sample size especially in various grades of fatty liver is the first limitation of the present study. Liver biopsy is the gold standard of assessing fatty liver but present study used ultrasonographic imaging studies for assessing grades of fatty liver which is another limitation of this study.

\section{Conclusion}

Rosuvastatin in addition to the hypocaloric diet might be an effective alternative treatment for hepatic steatosis. Although, rosuvastatin improved NFS and BARD scores but its effect on NASH and advanced fibrosis needs to be elucidated and validated further. The present study has reported that rosuvastatin treatment reduced the levels of serum periostin for the first time to the best of our knowledge. Furthermore, reversal of fatty infiltration is positively associated with the downregulation of serum periostin and upregulation of adiponectin after 6 months of rosuvastatin treatment which needs to be validated and elaborated in further large studies.

\section{Source of funding}

Sun Pharma, India provided partial assistance in the form of stipend under the joint collaboration for Ph.D. program with Jamia Hamdard, New Delhi, India.

\section{Conflict of interest declaration}

None.

\section{Acknowledgments}

We express special thanks to Dr. R.M. Pandey Professor \& Head, Department of Biostatistics, AIIMS, New Delhi for assistance in statistical analysis. We are also thankful to the nursing and pathology staff of Department of Medicine and Department of Radiology, Hamdard Institute of Medical Sciences \& Research, Jamia Hamdard, New Delhi, India for providing expert clinical assistance.

\section{References}

1. Calzadilla Bertot L, Adams LA. The natural course of non-alcoholic fatty liver disease. Int J Mol Sci. 2016;17:774.

2. Hyogo H, Tazuma S, Arihiro K, Iwamoto K, Nabeshima Y, Inoue M, et al. Efficacy of atorvastatin for the treatment of nonalcoholic steatohepatitis with dyslipidemia. Metabolism. 2008;57:1711-1718.

3. Pastori D, Polimeni L, Baratta F, Pani A, Del Ben M, Angelico F. The efficacy and safety of statins for the treatment of non-alcoholic fatty liver disease. Dig Liver Dis. 2015;47:4-11.

4. McTaggart F. Comparative pharmacology of rosuvastatin. Atheroscler Suppl. 2003;4:9-14.

5. Zhu JZ, Zhu HT, Dai YN, Li CX, Fang ZY, Zhao DJ, et al. Serum periostin is a potential biomarker for non-alcoholic fatty liver disease: a case-control study. Endocrine. 2016;51:91-100.
6. Lu Y, Liu X, Jiao Y, Xiong X, Wang E, Wang X, et al. Periostin promotes liver steatosis and hypertriglyceridemia through downregulation of PPAR $\alpha$. J Clin Invest. 2014;124:3501-3513.

7. Huang Y, Liu W, Xiao H, Maitikabili A, Lin O, Wu T, et al. Matricellular protein periostin contributes to hepatic inflammation and fibrosis. Am J Pathol. 2015; $185: 786-797$

8. Amara S, Lopez K, Banan B, Brown SK, Whalen M, Myles E, et al. Synergistic effect of pro-inflammatory TNF $\alpha$ and IL-17 in periostin mediated collagen deposition: potential role in liver fibrosis. Mol Immunol. 2015;64:26-35.

9. Dandan L, Daguo W. Effects of rosuvastatin on expression of periostin protein in rats with acute myocardial infarction. Chongqing Med. 2013;11:.

10. World Medical Association. World Medical Association Declaration of Helsinki: ethical principles for medical research involving human subjects. JAMA. 2013;310:2191.

11. von Elm E, Altman DG, Egger M, Pocock SJ, Gøtzsche PC, Vandenbroucke JP. STROBE Initiative. The strengthening the reporting of observational studies in epidemiology (STROBE) statement: guidelines for reporting observational studies. Lancet. 2007;370(9596):1453-1457.

12. Roza AM, Shizgal HM. The Harris Benedict equation reevaluated: resting energy requirements and the body cell mass. Am J Clin Nutr. 1984;40:168-182.

13. Matthews D, Hosker J, Rudenski A, Naylor BA, Treacher DF, Turner RC. Homeostasis model assessment: insulin resistance and $\beta$-cell function from fasting plasma glucose and insulin concentrations in man. Diabetologia. 1985;28:412-419.

14. Scatarige JC, Scott WW, Donovan PJ, Siegelman SS, Sanders RC. Fatty infiltration of the liver: ultrasonographic and computed tomographic correlation. J Ultrasound Med. 1984;3:9-14.

15. Gao X, Fan JG. Diagnosis and management of non-alcoholic fatty liver disease and related metabolic disorders: consensus statement from the study group of liver and metabolism, Chinese Society of Endocrinology. J Diabetis. 2013;5 (December (4)):406-415.

16. gulo P, Hui JM, Marchesini G, Bugianesi E, George J, Farrell GC, et al. The NAFLD fibrosis score: a noninvasive system that identifies liver fibrosis in patients with NAFLD. Hepatology. 2007;45:846-854.

17. Harrison SA, Oliver D, Arnold HL, Gogia S, Neuschwander-Tetri BA Development and validation of a simple NALFD clinical scoring system for identifying patient without advanced disease. Gut. 2008;57:1441-1447.

18. Souza Marinho T, Kawasaki A, Bryntesson M, Souza-Mello V, Barbosa-da-Silva S, Aguila MB, Mandarim-de-Lacerda CA. Rosuvastatin limits the activation of Hepatic stellate cells in diet-induced obese mice. Hepatol Res. 2016;47(9):92894010.1111/hepr.12821.

19. Mahalwar R, Khanna D. Pleiotropic antioxidant potential of rosuvastatin in preventing cardiovascular disorders. Eur J Pharmacol. 2013;711:57-62.

20. Antonopoulos S, Mikros S, Mylonopoulou M, Kokkoris S, Giannoulis G. Rosuvastatin as a novel treatment of non-alcoholic fatty liver disease in hyperlipidemic patients. Atherosclerosis. 2006;184:233-234.

21. Kargiotis K, Athyros VG, Giouleme O, Katsiki N, Katsiki E, Anagnostis P, et al. Resolution of non-alcoholic steatohepatitis by rosuvastatin monotherapy in patients with metabolic syndrome. World J Gastroenterol. 2015;21:7860.

22. Nakahara T, Hyogo H, Kimura Y, Ishitobi T, Arihiro K, Aikata H, et al. Efficacy of rosuvastatin for the treatment of non-alcoholic steatohepatitis with dyslipidemia: an open-label, pilot study. Hepatol Res. 2012;42:1065-1072.

23. Calderon RM, Cubeddu LX, Goldberg RB, Schiff ER. Statins in the treatment of dyslipidemia in the presence of elevated liver aminotransferase levels: a therapeutic dilemma. Mayo Clin Proc. 2010;85349-85356.

24. McKenney JM. Efficacy and safety of rosuvastatin in treatment of dyslipidemia. Am J Health Syst Pharm. 2005;62:1033-1047.

25. Arnaboldi L, Corsini A. Could changes in adiponectin drive the effect of statins on the risk of new-onset diabetes: the case of pitavastatin. Atheroscler Suppl. 2015:16:1-27.

26. Athyros VG, Alexandrides TK, Bilianou H, Mikhailidis DP, Mantzoros C. The use of statins alone, or in combination with pioglitazone and other drugs, for the treatment of non-alcoholic fatty liver disease/non-alcoholic steatohepatitis and related cardiovascular risk. An expert panel statement. Metabolism. 2017;71(June):17-32.

27. Luo B, Li B, Wang W, Liu X, Liu X, Xia Y, et al. Rosuvastatin alleviates diabetic cardiomyopathy by inhibiting NLRP3 inflammasome and MAPK pathways in a type 2 diabetes rat model. Cardiovasc Drugs Ther. 2014;28:33-43.

28. Luo Y, Qu H, Wang H, Wei H, Wu J, Duan Y, et al. Plasma periostin levels are increased in Chinese subjects with obesity and type 2 diabetes and are positively correlated with glucose and lipid parameters. Mediators Inflamm. 2016;23:2016

29. Snider P, Hinton RB, Moreno-Rodriguez RA, Wang J, Rogers R, Lindsley A, et al. Periostin is required for maturation and extracellular matrix stabilization of noncardiomyocyte lineages of the heart. Circ Res. 2008;102:752-760.

30. Ma YX, Li WH, Xie Q. Rosuvastatin inhibits TGF-1 expression and alleviates myocardial fibrosis in diabetic rats. Pharmazie. 2013;68:355-358.

31. Harrison SA, Fecht W, Brunt EM, Neuschwander-Tetri BA. Orlistat for overweight subjects with nonalcoholic steatohepatitis: a randomized, prospective trial. Hepatology. 2009;491:80-86.

32. Finelli C, Tarantino G. What is the role of adiponectin in obesity related non alcoholic fatty liver disease. World J Gastroenterol. 2013;19:802-812. 